Jani Onninen, Department of Mathematics, University of Jyväskylä, P.O.

Box 35, Fin-40351 Jyväskylä, Finland. e-mail: jaonnine@math.jyu.fi

\title{
DIFFERENTIABILITY OF MONOTONE SOBOLEV FUNCTIONS
}

\begin{abstract}
We establish a sharp integrability condition on the partial derivatives of a weakly monotone Sobolev function to guarantee differentiability almost everywhere.
\end{abstract}

\section{Introduction}

It is well known that if a function $u$ belongs to the Sobolev class $W^{1, p}(\Omega)$, $\Omega \subset \mathbb{R}^{n}$, for some $p>n$, then $u$ is differentiable almost everywhere in $\Omega$. This was proved by Cesari [2] for $n=2$ and by Calderón [1] for general $n$. Here $W^{1, p}(\Omega)$ consists of the functions in $\Omega$ which, together with their first order weak partial derivatives, are $p$-integrable. In 1981, Stein showed that this condition can be sharpened to a very precise integrability condition. He proved that if $u \in W^{1,1}(\Omega)$ is a function whose weak partial derivatives belong to the Lorentz space $L^{n, 1}(\Omega)$, then $u$ is differentiable almost everywhere. The notion of the Lorentz space $L^{p, q}(\Omega)$ was first introduced in [13].

In this paper we address the following question. What are the minimal integrability conditions on the partial derivatives of a homeomorphic Sobolev mapping $f \in W^{1,1}\left(\Omega, \mathbb{R}^{n}\right)$ needed to guarantee differentiability almost everywhere? Here $W^{1,1}\left(\Omega, \mathbb{R}^{n}\right)$ consists of the mappings of $\Omega$ into $\mathbb{R}^{n}$ whose coordinate functions belong to $W^{1,1}(\Omega)$. By the above result of Stein's, it naturally suffices to assume that $|\nabla f| \in L^{n, 1}(\Omega)$. However, less is needed. It suffices to assume that $|\nabla f|$ is $p$-integrable, for some $p>n-1$, whereas $|\nabla f| \in L^{n-1}(\Omega)$ is not sufficient when $n>2$. This was proved by Väisälä [19]. Väisälä's approach is itself an $n$-dimensional version of a technique used by Gehring and Lehto [5] to show that a planar homeomorphism with integrable partial derivatives is differentiable a.e. Recall that each function $u \in W^{1,1}(\Omega)$

Key Words: Differentiability, weakly monotonicity and Lorentz spaces

Mathematical Reviews subject classification: 26B35, 46E35

Received by the editors September 19, 2000

* The author was partially supported by the Academy of Finland, project 60127 
is differentiable a.e. when $\Omega \subset \mathbb{R}$. Because $L^{1,1}(\Omega)=L^{1}(\Omega)$, the results of Stein, Gehring and Lehto, and Väisälä suggest that the natural assumption should be that $|\nabla f| \in L^{n-1,1}(\Omega)$. We will show in this paper that this condition guarantees differentiability almost everywhere even for a larger class of mappings than the class of homeomorphisms. This is the class of weakly monotone mappings.

Monotonicity for a continuous function $u$ in a domain $\Omega \subset \mathbb{R}^{n}$ simply means that

$$
\sup _{x \in B} u(x) \leq \sup _{x \in \partial B} u(x) \text { and } \inf _{x \in B} u(x) \geq \inf _{x \in \partial B} u(x)
$$

for every ball $B \subset \Omega$. Roughly speaking, monotone functions satisfy the maximum and minimum principles in $\Omega$. This definition of monotone function is due to Lebesgue [12].

Definition 1.1. A real valued function $u \in W^{1,1}(\Omega)$ is said to be weakly monotone if for every ball $B \subset \Omega$ and all constants $m \leq M$ such that

$$
v:=(u-m)^{-}+(u-M)^{+} \in W_{0}^{1,1}(B),
$$

we have $m \leq u(x) \leq M$ for almost every $x \in B$.

Moreover, we say that $f \in W^{1,1}\left(\Omega, \mathbb{R}^{m}\right)$ is weakly monotone if its coordinate functions are weakly monotone.

Weakly monotone functions were introduced by Manfredi, [15]. They form a generalization of monotone functions in the sense of Lebesgue: a continuous function $u \in W^{1,1}(\Omega)$ is weakly monotone if and only if $u$ satisfies the conditions in (1). Especially, if $f: \Omega \rightarrow \Omega^{\prime}$ is a homeomorphic mapping of the class $W^{1,1}\left(\Omega, \mathbb{R}^{n}\right)$, then $f$ is a weakly monotone mapping. However is it not always true that weakly monotone functions are continuous (see [15, Example 2.1]). Many solutions to partial differential equations are weakly monotone. For example, if $0<\alpha(x) \leq \beta<\infty$ a.e. in $\mathbb{R}^{2}$, where $\alpha$ is a measurable function and $\beta$ is a constant, then each $W^{1,2}$-solution to $\operatorname{div} A(x) \nabla u(x)=0$ for

$$
A(x)=\left(\begin{array}{cc}
1 & 0 \\
0 & \alpha(x)
\end{array}\right)
$$

are weakly monotone (see [11]).

Now we can formulate our main theorem.

Theorem 1.2. (i) Suppose that $u \in W^{1,1}(\Omega)$ is a weakly monotone function whose weak partial derivatives are in $L^{n-1,1}(\Omega)$. Then $u$ is differentiable a.e. (ii) Suppose $q>1$. Then there is a weakly monotone function $v \in W^{1,1}(B(0,1))$ such that $|\nabla v| \in L^{n-1, q}(B(0,1))$ and $v$ is not differentiable anywhere. 
Here $L^{n, q}(\Omega)$ is the Lorenz space (see Section 2) and

$$
L^{p}(\Omega) \subset L^{n-1,1}(\Omega) \subset L^{n-1, q}(\Omega) \subset L^{n-1}(\Omega)
$$

locally, if $p>n-1$ and $1<q<n-1$ (see [20, Lemma 1.8.13]). Furthermore the above inclusions are strict.

One can prove that the following inclusion is true.

$$
\left\{u \in W^{1,1}(\Omega): \int_{\Omega}|\nabla u|^{n-1} \log ^{n-2+\epsilon}(e+|\nabla u|)<\infty, \epsilon>0\right\} \subset L^{n-1,1}(\Omega) .
$$

To illustrate the significance of Theorem 1.2 in the case of homeomorphisms for a reader not familiar with Lorentz spaces we give the following example that follows from part $(i)$ and the proof of part $(i i)$.

Example 1.3. Let $u: \Omega \rightarrow \Omega^{\prime}$ be a homeomorphism in class $W^{1,1}\left(\Omega, \mathbb{R}^{n}\right)$ where $\Omega \subset \mathbb{R}^{n}$. Then the condition $\int_{\Omega}\left|\nabla u_{i}\right|^{n-1} \log ^{\alpha}\left(e+\left|\nabla u_{i}\right|\right)<\infty$ for all $i=1, \ldots, m$ guarantees differentiability a.e. if $\alpha>n-2$ but not if $\alpha \leq n-2$.

\section{Preliminaries}

The notation used in this paper is standard. The symbol $\Omega$ denotes an open subset of $\mathbb{R}^{n}, n \geq 2$. $B^{n}(x, r)$ and $B(x, r)$ will denote an $n$-dimensional ball centered at $x$ with the radius $r$. Spheres will be denoted by $S^{n-1}(x, r)$. The Lebesgue measure of a set $E$ will be denoted by $|E|$. The characteristic function of a set $E$ is $\chi_{E}$. If $u: E \rightarrow \mathbb{R}$ is an integrable function on a set $E$ with $0<|E|<\infty$, we write $u_{E}=f_{E} u(x) d x=\frac{1}{|E|} \int_{E} u(x) d x . C(a, b)$ will denote a positive constant that depends only on $a, b$. The value of $C(a, b)$ is not necessary the same at each occurrence; it may vary even within a line.

The gradient $\nabla u$ is understood in the distributional sense. We use $W^{1,1}(\Omega)$ to denote the usual Sobolev space on $\Omega$ consisting of functions $u$ such that both $u \in L^{1}(\Omega)$ and $|\nabla u| \in L^{1}(\Omega)$. The space is equipped with the norm $\|u\|_{W^{1,1}(\Omega)}=\|u\|_{L^{1}(\Omega)}+\|\mid \nabla u\|_{L^{1}(\Omega)}$. The space $W_{0}^{1,1}(\Omega)$ denotes the completion of $C_{0}^{\infty}(\Omega)$ in $W^{1,1}(\Omega)$. $W^{1,1}\left(\Omega, \mathbb{R}^{m}\right)$ denotes the class of mappings $u: \Omega \rightarrow \mathbb{R}^{m}$ such that the coordinate functions belong to the space $W^{1,1}(\Omega)$.

Let $u$ be a $\mu$-measurable function defined on $X$, where $(X, \mu)$ is a measure space. We denote by $\omega$ the distribution function of $u$, namely, for $t \geq 0$ we set

$$
\omega(t)=\mu(\{x \in X: u(x)>t\}) .
$$

Then we define the the non-increasing rearrangement $u^{*}$ of $u$ by setting

$$
u^{*}(s)=\inf \{t \geq 0: \omega(t) \leq s\} .
$$


It is well known that if $u$ and $v$ are two $\mu$-measurable functions on $X$, then

$$
\int_{X}|u(x) v(x)| d \mu(x) \leq \int_{0}^{\mu(X)} u^{*}(s) v^{*}(s) d s .
$$

The inequality (2) is due to Hardy and Littlewood [7]. It follows immediately from the definition of $u^{*}(s)$ that

$$
u^{*}(\omega(t)) \leq t
$$

The Lorentz space $L^{p, q}(X), 1 \leq p<\infty, 1 \leq q \leq \infty$, is defined as the class of all measurable functions on $\Omega$ for which the norm

$\|u\|_{L^{p, q}(X)}:= \begin{cases}\left(\int_{0}^{\mu(X)}\left[t^{\frac{1}{p}} u^{*}(t)\right]^{q} \frac{d t}{t}\right)^{\frac{1}{q}}=\left(p \int_{0}^{\infty} s^{q-1}[\omega(s)]^{\frac{q}{p}} d s\right)^{\frac{1}{q}} & 1 \leq q<\infty \\ \sup _{s>0} s[\omega(s)]^{\frac{1}{p}} & q=\infty\end{cases}$

is finite. For further details about the Lorentz space, see e.g. [17] or [20].

\section{The Oscillation Lemma}

The aim of this section is to establish an oscillation estimate for weakly monotone functions in the Lorentz space $L^{n-1,1}$. The elements in the Sobolev space $W^{1,1}(\Omega)$ are equivalence classes of functions which agree almost everywhere in $\Omega$. In order to study the fine properties of a function $u \in W^{1,1}(\Omega)$, it is convenient to use the representative $\tilde{u}$, defined by the formula

$$
\tilde{u}(x)=\lim _{r \rightarrow 0} \sup f_{B(x, r)} u(z) d z .
$$

It is well known that if $v: \mathbb{R}^{n} \rightarrow \mathbb{R}$ is locally integrable, then

$$
\lim _{r \rightarrow 0} f_{B(x, r)}|v(x)-v(z)| d z=0
$$

for almost all $x \in \mathbb{R}^{n}$. Hence $\tilde{u}$ is a Borel measurable representative of $u$. The function $\tilde{u}$ is a natural representative of $u$, for example if we know that $v=u$ a.e. and $v$ is a continuous function, then $v \equiv \tilde{u}$.

Now we can formulate our oscillation lemma.

Lemma 3.1. Let $u \in W^{1,1}(B(a, 2 r))$ be a weakly monotone function such that $|\nabla u| \in L^{n-1,1}(B(a, 2 r))$. Then for all $x, y \in B(a, r)$

$$
|\tilde{u}(x)-\tilde{u}(y)| \leq C(n) r^{-\frac{1}{n-1}}\|\nabla u\|_{L^{n-1,1}(B(a, 2 r))} .
$$


If $|\nabla u| \in L^{n, 1}(B(a, 2 r))$, then we have the following oscillation lemma. This Lemma holds without the assumption that $u$ is weakly monotone. It is essentially due to Stein [16]. For the convenience of the reader we give a complete proof below; see [4, Inequalities (9.6) and (5.7)] and [10] for alternate proofs.

Lemma 3.2. Let $u \in W^{1,1}(B(x, R))$ be a function so that $|\nabla u| \in L^{n, 1}(B(x, R))$. Then there exists a constant $C(n)$ such that for all $y \in B(x, R)$

$$
|\tilde{u}(x)-\tilde{u}(y)| \leq C(n)\|\nabla u\|_{L^{n, 1}(B(x,|x-y|))}
$$

Proof. Fix $y \in B(x, R)$ and denote $r_{i}=\left(\frac{|x-y|}{4}\right) 2^{-|i|}$ for all $i \in \mathbb{Z}$.

Let $x_{0}=\frac{x+y}{2}$ and define recursively

$$
x_{i}=x_{i-1}+r_{i-1} \frac{x-y}{|x-y|} \text { for } i>0
$$

and

$$
x_{i}=x_{i+1}-r_{i+1} \frac{x-y}{|x-y|} \text { for } i<0 .
$$

Then the balls $B_{i}=B\left(x_{i}, r_{i}\right) \subset B(x,|x-y|)$ and the points $x_{i}$ satisfy

$$
\begin{aligned}
& \text { (i) } B\left(x_{i}, r_{i}\right) \subset B\left(x_{i+1}, 4 r_{i+1}\right) \text { when } i \geq 0, \\
& B\left(x_{i}, r_{i}\right) \subset B\left(x_{i-1}, 4 r_{i-1}\right) \text { when } i \leq 0, \\
& \text { (ii) } \sum_{i} \chi_{B_{i}} \leq 2, \\
& \text { (iii) } x_{i} \rightarrow x \text { as } i \rightarrow \infty, \\
& x_{i} \rightarrow y \text { as } i \rightarrow-\infty, \\
& \left(i v\left|x_{i}-x\right| \leq 2 r_{i} \text { when } i \geq 0\right. \text { and } \\
& \left|x_{i}-y\right| \leq 2 r_{i} \text { when } i \leq 0 .
\end{aligned}
$$

From properties $(i i i)$ and $(i v)$ it follows that $u_{B_{i}} \rightarrow \tilde{u}(x)$ as $i \rightarrow \infty$ and $u_{B_{i}} \rightarrow \tilde{u}(y)$ as $i \rightarrow-\infty$. Because

$$
\tilde{u}(x)=u_{B_{0}}-\sum_{i=0}^{\infty}\left(u_{B_{i}}-u_{B_{i+1}}\right) \text { and } \tilde{u}(y)=u_{B_{0}}-\sum_{i=0}^{-\infty}\left(u_{B_{i}}-u_{B_{i-1}}\right)
$$

we have

$$
|\tilde{u}(x)-\tilde{u}(y)| \leq \sum_{i=0}^{\infty}\left|u_{B_{i}}-u_{B_{i+1}}\right|+\sum_{i=0}^{-\infty}\left|u_{B_{i}}-u_{B_{i-1}}\right|
$$


Applying the Poincaré inequality we obtain

$$
\begin{aligned}
|\tilde{u}(x)-\tilde{u}(y)| & \leq C(n) \sum_{i=-\infty}^{\infty} r_{i}^{1-n} \int_{B_{i}}|\nabla u| d z \\
& =C(n) \sum_{i=-\infty}^{\infty} r_{i}^{1-n} \int_{\left\{(z, t) \in B_{i} \times(0, \nabla u(z))\right\}} d(z, t)
\end{aligned}
$$

Let $\omega$ be the distribution function of $|\nabla u|$; i.e., $\omega(t)=\mid\{y \in B(x,|x-y|)$ : $|\nabla u(y)|>t\} \mid$ for all $t>0$. Next we split the set $\left\{(z, t) \in B_{i} \times(0, \nabla u(z))\right\}=: E_{i}$ into $\left\{(z, t) \in E_{i}: \omega(t) \geq r_{i}^{n}\right\}$ and $\left\{(z, t) \in E_{i}: \omega(t)<r_{i}^{n}\right\}$. For $i \geq 0$ from properties $(i i)$ and $(i v)$ it follows that

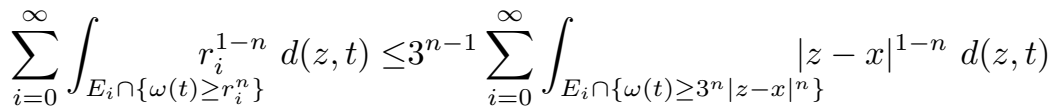

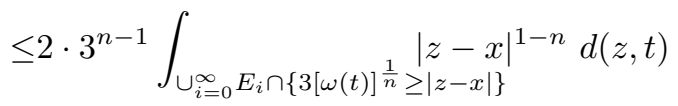

$$
\begin{aligned}
& \leq 2 \cdot 3^{n-1} \int_{0}^{\infty} \int_{B\left(x, 3[\omega(t)]^{\frac{1}{n}}\right)}|z-x|^{n-1} d z d t \\
& \leq C(n) \int_{0}^{\infty}[\omega(t)]^{\frac{1}{n}} d t
\end{aligned}
$$

Similarly for $i \leq 0$ we have

$$
\sum_{i=-\infty}^{0} \int_{E_{i} \cap\left\{\omega(t) \geq r_{i}^{n}\right\}}^{r_{i}^{1-n}} d(z, t) \leq C(n) \int_{0}^{\infty}[\omega(t)]^{\frac{1}{n}} d t
$$

For the $\left\{(z, t) \in E_{i}: \omega(t)<r_{i}^{n}\right\}$-part we have that

$$
\begin{aligned}
\sum_{i=-\infty}^{\infty} \int_{E_{i} \cap\left\{\omega(t)<r_{i}^{n}\right\}} r_{i}^{1-n} d(z, t) & \leq \int_{\cup_{i=-\infty}^{\infty} E_{i}}[\omega(t)]^{\frac{1-n}{n}} d(z, t) \\
& \leq \int_{B(x,|x-y|)}|\nabla(z)|[\omega(|\nabla(z)|)]^{\frac{1-n}{n}} d z \\
& \leq \int_{0}^{|B(x,|x-y|)|}|\nabla(t)|^{*} t^{\frac{1-n}{n}} d t .
\end{aligned}
$$

The last inequality follows from (2) and (3). We obtain the desired inequality (4) from (5), (6), (7) and (8). 
We will deduce Lemma 3.1 from Lemma 3.2. For this we will employ an approximation argument. It is well known that we can approximate Sobolev functions by smooth functions. If a Sobolev function is weakly monotone, then it is surprising that the approximations can be in fact chosen to be "almost" monotone. Let us give a precise statement of the result that is due to Iwaniec, Koskela and Onninen [8].

Lemma 3.3. Let $u \in W^{1,1}(B(a, R))$ be a weakly monotone function and $r<R$. Suppose that $\delta>0$. Then there exists a number $j_{0} \in \mathbb{N}$ and a sequence $\left\{u_{j}\right\}_{j=1}^{\infty} \subset C^{\infty}(\Omega)$ such that $u_{j} \rightarrow u$ in $W_{\text {loc }}^{1,1}(B(a, R))$ and $u_{j}\left(x_{0}\right) \rightarrow u\left(x_{0}\right)$ for all Lebesgue points $x_{0} \in B(a, R)$. Furthermore for all Lebesgue points $x_{0}, y_{0} \in B(a, r)$ for all $j \geq j_{0}$ and every $r \leq t \leq R$

$$
\left|u_{j}\left(x_{0}\right)-u_{j}\left(y_{0}\right)\right| \leq \operatorname{osc}\left(u_{j}, S^{n-1}(a, t)\right)+2 \delta .
$$

Now we are ready to prove lemma 3.1.

Proof of Lemma 3.1 Fix $t \in(r, 2 r)$. Applying Lemma 3.2 on spheres for the function $u_{j} \in C^{\infty}(B(a, 2 r))$ at (9) we have

$$
\left|u_{j}\left(x_{0}\right)-u_{j}\left(y_{0}\right)\right| \leq C(n)|| \nabla u_{j} \|_{L^{n-1,1}\left(S^{n-1}(a, t)\right)}+2 \delta
$$

for all $t \in(r, 2 r)$ and all Lebesgue points $x_{0}, y_{0} \in B(a, r)$. To see that Lemma 3.2 applies to our situation notice that $u_{j}$ is smooth and any pair of points on $S^{n-1}(a, t)$ can be joined using a chain of no more than $n^{2}$ spherical caps, each of which is bi-Lipschitz (with a uniform constant) equivalent to $B^{n-1}\left(0, \frac{t}{2}\right)$. Integrating over the interval $r<t<2 r$ and dividing both sides by $\mathrm{r}$, we obtain

$$
\begin{aligned}
\left|u_{j}\left(x_{0}\right)-u_{j}\left(y_{0}\right)\right| \leq & \frac{C(n)}{r} \int_{r}^{2 r} \int_{0}^{\infty}\left[\mathcal{H}^{n-1}\left(\left\{x \in S^{n-1}(a, t):\left|\nabla u_{j}\right|>s\right\}\right)\right]^{\frac{1}{n-1}} d s d t \\
& +2 \delta \\
= & \frac{C(n)}{r} \int_{0}^{\infty} \int_{r}^{2 r}\left(\int_{S^{n-1}(a, t)} \chi_{\left\{\left|\nabla u_{j}\right|>s\right\}}(y) d \mathcal{H}^{n-1}(y)\right)^{\frac{1}{n-1}} d s d t \\
& +2 \delta
\end{aligned}
$$


Then, applying Hölder's inequality, we obtain

$$
\begin{aligned}
\left|u_{j}\left(x_{0}\right)-u_{j}\left(y_{0}\right)\right| \leq & \frac{C(n)}{r} \int_{0}^{\infty} r^{\frac{n-2}{n-1}}\left(\int_{r}^{2 r} \int_{S^{n-1}(a, t)} \chi_{\left\{\left|\nabla u_{j}\right|>s\right\}}(y) d \mathcal{H}^{n-1}(y) d t\right)^{\frac{1}{n-1}} d s \\
& +2 \delta \\
\leq & C(n) r^{-\frac{1}{n-1}} \int_{0}^{\infty}\left(\int_{B(a, 2 r)} \chi_{\left\{\left|\nabla u_{j}\right|>s\right\}}(y) d y\right)^{\frac{1}{n-1}} d s+2 \delta \\
= & C(n) r^{-\frac{1}{n-1}}\left\|\nabla u_{j}\right\|_{L^{n-1,1}(B(a, 2 r))}+2 \delta
\end{aligned}
$$

By letting $j \rightarrow \infty$ and $\delta \rightarrow 0$ we conclude that

$$
\left|u\left(x_{0}\right)-u\left(y_{0}\right)\right| \leq\left. C(n) r^{-\frac{1}{n-1}}|| \nabla u\right|_{L^{n-1,1}(B(a, 2 r))} .
$$

Let $x \in B(a, r)$ and $s<r-|x-a|$. Then

$$
\begin{aligned}
\left|f_{B(x, s)} u(z) d z-u\left(x_{0}\right)\right| & \leq f_{B(x, s)}\left|u(z)-u\left(x_{0}\right)\right| d z \\
& \leq C(n) r^{-\frac{1}{n-1}}\|\nabla u\|_{L^{n-1,1}(B(a, 2 r))}
\end{aligned}
$$

which gives

$$
\left|\tilde{u}(x)-u\left(x_{0}\right)\right| \leq C(n) r^{-\frac{1}{n-1}}|| \nabla u \|_{L^{n-1,1}(B(a, 2 r))}
$$

and so

$$
|\tilde{u}(x)-\tilde{u}(y)| \leq\left. C(n) r^{-\frac{1}{n-1}}|| \nabla u\right|_{L^{n-1,1}(B(a, 2 r))}
$$

for all $x, y \in B(a, r)$.

\section{Proof of Theorem 1.2}

In the proof we will use the following lemma that goes back to Stepanov [18] (see also [14]).

Lemma 4.1. A function $u: \Omega \rightarrow \mathbb{R}$ is differentiable a.e. if and only if

$$
\limsup _{y \rightarrow x} \frac{|u(x)-u(y)|}{|x-y|}<\infty \text { a.e. }
$$


Now $(i)$ of Theorem 1.2 follows from Lemma 3.1 and Lemma 4.1 if

$$
\limsup _{r \rightarrow 0} r^{-\frac{n}{n-1}}\|\nabla u\|_{L^{n-1,1}(B(a, r))}
$$

is finite for almost every $a \in \Omega$.

The following lemma is due to Stein [16]. It is a consequence of the standard estimate for the Hardy-Littlewood maximal function: If $g \in L^{1}\left(\mathbb{R}^{n}\right)$, then

$$
\left|\left\{x \in \mathbb{R}^{n}: \sup _{r>0} f_{B(x, r)}|g(y)| d y>t\right\}\right| \leq \frac{C(n)}{t} \int_{\mathbb{R}^{n}}|g(x)| d x .
$$

Lemma 4.2. Suppose that $f \in L^{p, 1}(\Omega), 1<p<\infty$. Then for a.e. $x \in \Omega$

$$
\limsup _{r \rightarrow 0} r^{-\frac{n}{p}}\|f\|_{L^{p, 1}(B(x, r))}<\infty .
$$

For the convenience of the reader we give a short proof below; Stein's proof in [16] consists of a list of references to various estimates.

PROOF. For $x \in \Omega$ let $M_{p}(f)(x)=\sup _{r>0} r^{-\frac{n}{p}}\|f\|_{L^{p, 1}(B(x, r))}$. We will show that

$$
\left\|M_{p}(f)\right\|_{L^{p, \infty}(\Omega)} \leq C\|f\|_{L^{p, 1}(\Omega)} .
$$

If we take inequality (11) as known, then

$$
\left|\left\{x \in \Omega: \limsup _{r \rightarrow 0} r^{-\frac{n}{p}}\|f\|_{L^{p, 1}(B(x, r))}=\infty\right\}\right| \leq \limsup _{k \rightarrow \infty} \frac{C\|f\|_{L^{p, 1}(\Omega)}^{p}}{k^{p}}=0
$$

as desired.

We observe that it suffices to establish inequality (11) when $f=\sum_{i=1}^{h} c_{i} \chi_{A_{i}}$ is a non-negative simple function (see [20, Proposition 1.8.4]). We can assume that $c_{1}>c_{2}>\cdots>c_{h}, A_{i} \cap A_{j}=\emptyset$, when $i \neq j$ and $\left|A_{i}\right|<\infty$ for all $i \in\{1, \ldots h\}$. Set $c_{h+1}=0$. Then

$$
\begin{aligned}
\|f\|_{L^{p, 1}(\Omega)} & =\sum_{i=1}^{h} \int_{c_{i+1}}^{c_{i}}|\{x \in \Omega: f(x)>t\}|^{\frac{1}{p}} d t \\
& =\sum_{i=1}^{h}\left(c_{i}-c_{i+1}\right)\left|\cup_{j=1}^{i} A_{j}\right|^{\frac{1}{p}} .
\end{aligned}
$$

Because $f=\sum_{i=1}^{h} c_{i} \chi_{A_{i}}=\sum_{i=1}^{h}\left(c_{i}-c_{i+1}\right) \chi_{\cup_{j=1}^{i} A_{j}}$, we obtain

$$
\begin{aligned}
\left\|M_{p}(f)\right\|_{L^{p, \infty}(\Omega)} & \leq \sum_{i=1}^{h}\left(c_{i}-c_{i+1}\right)\left\|M_{p}\left(\chi_{\cup_{j=1}^{i} A_{j}}\right)\right\|_{L^{p, \infty}(\Omega)} \\
& \leq C \sum_{i=1}^{h}\left(c_{i}-c_{i+1}\right)\left|\cup_{j=1}^{i} A_{j}\right|^{\frac{1}{p}} .
\end{aligned}
$$


The last inequality follows from

$$
M_{p}\left(\chi_{A}\right)(x)=\sup _{r>0} r^{-\frac{n}{p}}|A \cap B(x, r)|^{\frac{1}{p}}=\left(\sup _{r>0} r^{-n}|A \cap B(x, r)|^{n}\right)^{\frac{1}{p}}
$$

for arbitrary $A$ of finite measure and inequality (10). Now inequality (11) follows from (12) and (13).

(ii) Suppose that $q>1$. Then there is a radial function $f \in W^{1,1}\left(B^{n-1}(0,2)\right)$ such that $|\nabla f| \in L^{n-1, q}\left(B^{n-1}(0,2)\right)$ and $\lim _{r \rightarrow 0} \operatorname{ess} \sup \{f(x): x \in B(0, r)\}=\infty$. The existence of such a function follows from [3] or [9]. The set

$$
\left\{\left(x_{1}, \ldots, x_{n-1}\right) \in B^{n-1}(0,1): x_{i} \in \mathbb{Q}, i=1, \ldots, n-1\right\}
$$

is dense in $B^{n-1}(0,1)$. Denote those points $q_{1}, q_{2}, q_{3}, \ldots$ and define $g_{j}(x)=$ $2^{-j} f\left(x-q_{j}\right)$. Set $h_{k}(x)=\sum_{j=1}^{k} g_{j}(x)$ for all $x \in B^{n-1}(0,1)$. Because the space $W^{1,1}\left(B^{n-1}(0,1)\right)$ is a Banach space and for $j<k$

$$
\begin{aligned}
\left\|h_{k}-h_{j}\right\|_{W^{1,1}\left(B^{n-1}(0,1)\right)} & =\left\|\sum_{i=j+1}^{k} g_{i}\right\|_{W^{1,1}\left(B^{n-1}(0,1)\right)} \\
& \leq \sum_{i=j+1}^{k}\left\|g_{i}\right\|_{W^{1,1}\left(B^{n-1}(0,1)\right)} \\
& \leq\|f\|_{W^{1,1}\left(B^{n-1}(0,2)\right)} \sum_{i=j+1}^{k} 2^{-i}
\end{aligned}
$$

we have that the sequence $\left(h_{j}\right)_{j}$ converges to some $h$ in $W^{1,1}\left(B^{n-1}(0,1)\right)$. Furthermore $L^{n-1, q}\left(B^{n-1}(0,1)\right)$ is a Banach space [17, Chapter v, Theorem 3.22.] and thus the same argument as above shows that $|\nabla h| \in L^{n-1, q}\left(B^{n-1}(0,1)\right)$. Finally set $v\left(x_{1}, \ldots, x_{n}\right)=h\left(x_{1}, \ldots, x_{n-1}\right)$ for all $x \in B^{n}(0,1)$. It follows that the function $v$ is weakly monotone. Suppose $\hat{v}=v$ almost everywhere in $B^{n}(0,1)$, and fix $i \in \mathbb{N}$ and $z \in \mathbb{Q}$ such that $B^{n}\left(\left(q_{i}, z\right), r_{0}\right) \subset B^{n}(0,1)$ for some $r_{0}>0$. Then

$\lim _{r \rightarrow 0} \sup \left\{\hat{v}(x): x \in B^{n}\left(\left(q_{i}, z\right), r\right)\right\} \geq \lim _{r \rightarrow 0} \operatorname{ess} \sup \left\{g_{i}(x): x \in B^{n-1}\left(q_{i}, r\right)\right\}=\infty$

Because the set $\left\{x \in B^{n}(0,1): x_{i} \in \mathbb{Q}, i=1, \ldots, n\right\}$ is dense in $B^{n}(0,1)$, it follows that $\hat{v}$ is non-differentiable (even discontinuous) everywhere in $B^{n}(0,1)$.

In 1981, Stein [16] proved that if $f \in W^{1,1}(\Omega)$ and $|\nabla f| \in L^{n, 1}(\Omega)$, then $f$ is differentiable a.e. We can give an alternate proof of the first part of 
Theorem 1.2 based on this fact on spheres and some tools from nonlinear potential theory, along the lines of the argument in [19]. This approach is however technical and involves the notion of trace. Notice that our function $u$ need not be continuous.

Acknowledgement: We thank Pekka Koskela for important suggestions.

\section{References}

[1] A. P Calderón, On the differentiability of absolutely continuous functions, Riv. Mat. Univ. Parma., 2 (1951), 203-213.

[2] L. Cesari, Sulle funzioni assolutament continue in due variability, Ann. Scuola Norm. Sup. Pisa., 10 (1941), 91-101.

[3] A. Cianchi and L. Pick, Sobolev embeddings into BMO, VMO, and $L_{\infty}$, Ark. Mat., 36 (1998), 317-340.

[4] R. A. DeVore and R. C. Sharpley, Maximal functions measuring smoothness, Mem. Amer. Math. Soc., 47 (1984), no. 293.

[5] F. W. Gehring and O. Lehto, On the total differentiability of functions of a complex variable, Ann. Acad. Sci. Fenn. Ser. A I Math., 272 (1959), $1-9$.

[6] P. Hajłasz, and J. Malý, Approximation of nonlinear expressions involving gradient in Sobolev spaces, Preprint.

[7] G. H. Hardy, J. H. Littlewood and G. Pólya, Inequalities, Cambridge University Press, (1934).

[8] T. Iwaniec, P. Koskela J. and Onninen, Mappings of finite distortion: Monotonicity and continuity, Inventiones Mathematicae, to appear.

[9] J. Kauhanen, P. Koskela and J. Malý, (1999) On functions with derivatives in a Lorentz space, Manuscripta Math., 100, no. 1 (1999), 87-101.

[10] V. I. Kolyada, Estimates for rearrangements and embedding theorems, Math. USSR-Sb., 64, no. 1 (1988), 1-21.

[11] P. Koskela, J. J. Manfredi and E. Villamor, Regularity theory and traces of $\mathcal{A}$-harmonic functions, Trans. Amer. Math. Soc., 348, no. 2 (1996), $755-766$.

[12] H. Lebesgue, Sur le problème de Dirichlet, Rend. Circ. Palermo, 27 (1907), 371-402. 
[13] G. G. Lorentz, Some new functional spaces, Ann. of Math., 51 (1950), $37-55$.

[14] J. Malý, A simple proof of the Stepanov theorem on differentiability almost everywhere, Exposition. Math., 17 (1999), 59-61.

[15] J. Manfredi, Weakly Monotone Functions, The Journal of Geometric Analysis, 3 (1994), 393-402.

[16] E. M. Stein, Editor's note: The differentiability of functions in $\mathbb{R}^{n}$, Annals of Mathematics, 113 (1981), 383-385.

[17] E. M. Stein and G. Weiss, Introduction to Fourier Analysis on Euclidean Spaces, Princeton Univ. Press, (1971).

[18] V. Stepanoff, Sur les conditions de l'existence de la differentielle totale, Mat. Sb., 32 (1925), 511-526.

[19] J. Väisälä, Two new characterizations for quasiconformality, Ann. Acad. Sci. Fenn. Ser. A I Math., 362 (1965), 1-12.

[20] W. P. Ziemer, Weakly Differentiable Functions, Graduate Texts in Mathematics, 120, Springer-Verlag, (1989). 